\title{
EFECTO DEL AMONÍACO SOBRE EL CRECIMIENTO DE LA GAMITANA Colossoma macropomum (Cuvier, 1818) ${ }^{1}$
}

\author{
Rosa Ismiño ${ }^{2^{*}}$, Carlos Araujo-Lima ${ }^{3^{*}}$
}

\section{RESUMEN}

El efecto de la concentración de amoníaco sobre el crecimiento fue probado usando soluciones acuosas con concentraciones de 0.002 (control), 0.07 y $0.46 \mathrm{mg} / 1 \mathrm{de} \mathrm{NH}_{3}$ y un control. Los peces fueron mantenidos en tanques de 500 litros con estas soluciones por un período de 2 meses. No se observaron efectos significativos del amoníaco sobre el crecimiento de la gamitana en este período. Se concluye que la gamitana es una especie muy tolerante y que puede ser mantenida en concentraciones de amoníaco de $0.46 \mathrm{mg} / \mathrm{l}$ sin comprometer su crecimiento.

Palabras claves: Amoníaco, crecimiento, Colossoma macropomum

\begin{abstract}
The effect of ammonia concentration on the growth rate was tested at three levels: 0.002 (control); $0.07 ; 0.46 \mathrm{mg} / 1 \mathrm{NH}_{3}$. Fish were kept in 500 liter tanks with experimental water solution for 2 months. There was no effect of ammonia concentration up to $0.46 \mathrm{mg} / 1 \mathrm{NH}_{3}$ on the growth rate of Colossoma macropomum.
\end{abstract}

\section{INTRODUCCIÓN}

Entre los peces de agua dulce, los serrasalmidos están considerados como uno de los grupos más utilizados en acuicultura. La especie Colossoma macropomum fue adaptada con éxito para el cultivo en cautiverio, siendo la mas indicada para el policultivo, por su capacidad para aprovechar diferentes tipos de alimentos y por su rápido crecimiento.

1 Parte de la tesis de Maestría del primer autor

2 Instituto de Investigaciones de la Amazonía Peruana (IIAP). Apto. postal 784. Iquitos, Perú.

3 Instituto Nacional de Pesquisas Da Amazonia (INPA) Alameda Cosme Ferreira, 1756.69083000, Manaus, Brasil 
Dentro de los productos nitrogenados, el amoníaco es el producto final del catabolismo proteico de la mayoría de los organismos acuáticos y es un contaminante común en sistemas acuáticos, pudiendo ser extremadamente tóxico para los peces especialmente en $\mathrm{pH}$ altos y en sistemas con alta carga orgánica (Thurston et al; 1986), teniendo consecuencias negativas para el desarrollo y sobrevivencia de los peces (Jobling; 1994).

El efecto negativo de la toxicidad en organismos acuáticos es atribuido al amoniaco en su forma no ionizada $\left(\mathrm{NH}_{3}\right)$ (Wajsbrot et al; 1993). Exposiciones por debajo de los niveles tóxicos de amoníaco causan degeneración de la piel, daños en las branquias y los riñones (Tomasso et al., 1980), y reducción en la capacidad de transporte de hemoglobina (Sousa \& Meade, 1977). El amoníaco en su forma ionizada, y no ionizada, retardan el crecimiento y causan cambios histopatológicos en diferentes órganos.

La concentración letal del amoníaco $\left(\mathrm{NH}_{3}\right)$ citada para los peces varía de 0.2 a $2 \mathrm{mg} / \mathrm{l}$. La sensibilidad puede variar con el tamaño.

Este trabajo evalúa el impacto de la acumulación del amoníaco $\left(\mathrm{NH}_{3}\right)$ en el agua sobre el desarrollo de los peces en cultivo y su efecto en el crecimiento por un período de 60 días.

\section{MATERIAL Y MÉTODOS}

La metodología empleada fue estática y se observó las normas citadas por Sprague (1990) para procedimientos en ecotoxicología acuática.

\section{Animales Experimentales}

Inicialmente, se seleccionaron 30 peces de 43.5 a $198 \mathrm{~g}$ de peso, que fueron aclimatados en tanques de fibrocemento de 500 litros de capacidad, con 350 litros de agua, por un período de 5 días. La temperatura y el $\mathrm{pH}$ del agua variaron entre $24.4 \mathrm{y}$ $27.2^{\circ} \mathrm{C}$ y de 6 y 6.9 , respectivamente. Los peces recibieron alimentación en una tasa de $2 \%$ de la biomasa por día con calidad nutricional (24.5\% de proteína). Los tanques fueron equipados con filtros biológicos y la aireación fue constante. 


\section{Tratamiento experimental}

Al final del período de aclimatación, 6 tanques fueron preparados: 2 con tratamiento de control, otros 2 con bajas concentraciones de amoníaco de $0.07 \mathrm{mg} \mathrm{de} \mathrm{NH}_{3} \mathrm{mg} / \mathrm{l}$, y los 2 restantes con altas concentraciones de amoníaco $\left(0.46 \mathrm{mg} \mathrm{de} \mathrm{NH}_{3} \mathrm{mg} / \mathrm{l}\right)$. Se utilizó cloruro de amonio $\left(\mathrm{NH}_{4} \mathrm{CL}\right)$ como tóxico. Las concentraciones experimentales fueron definidas en relación a la temperatura y $\mathrm{pH}\left(28^{\circ} \mathrm{C}\right.$ y 8 , respectivamente). Los controles no recibieron adición de $\mathrm{NH}_{4} \mathrm{CL}$. Para obtener las concentraciones experimentales de $\mathrm{NH}_{3}$ se usó $30.13 \mathrm{~g} / \mathrm{l}$ y $200.87 \mathrm{~g} / 1$ de $\mathrm{NH}_{4} \mathrm{CL}$ para los tratamientos con bajas y altas concentraciones de amoníaco.

El estudio fue realizado en un período total de 60 días, se trabajó 5 peces por tanque. Las heces fueron sifoneadas dos veces al día. La concentración de amoníaco en los tanques tendió a aumentar debido a la excreción de los peces. Considerando el pH, la temperatura, la tasa de excreción y el volumen del tanque, la concentración de amoníaco $\left(\mathrm{NH}_{3}\right)$ sería de $0.0009 \mathrm{mg} / \mathrm{l}$. Este aumento es inferior a $1 \%$ de la concentración experimental. La temperatura, el $\mathrm{pH}$ y el $\mathrm{NH}_{4}$ fueron monitoreados diariamente con el fin de mantener las concentraciones experimentales de amoníaco $\left(\mathrm{NH}_{3}\right)$ en el sistema.

Los peces en estudio se marcaron con pequeñas cuentas en la región de la aleta dorsal después de pesarlos en la balanza electrónica, y fueron repuestos a sus respectivos tanques. Este procedimiento fue realizado al inicio y final del experimento. El crecimiento fue expresado como tasa de crecimiento específico ( $\mathrm{TCE}=\ln ($ Peso final)$\ln$ (Peso inicial)/Tiempo X 100). El efecto de las dos concentraciones de amoníaco sobre el crecimiento fue comparado con el tratamiento control, usando un análisis de covarianza. Como la tasa de crecimiento específica está en función de la potencia del peso del pez (Ricker, 1979; Elliot \& Hurley 1995), el peso fue usado como covariable en el análisis, y la concentración de amoníaco como factor.

\section{RESULTADOS}

Los 6 tanques presentaron un patrón semejante en la variación de temperatura y $\mathrm{pH}$ (Fig. 1). La temperatura tuvo una variabilidad máxima de $2.7^{\circ} \mathrm{C}\left(24.5 \mathrm{~A} 27.2^{\circ} \mathrm{C}\right)$ con un promedio de $25.9^{\circ} \mathrm{C}$ (D.P=0.6). Los valores de $\mathrm{pH}$ variaron de 6 a 6.9. $\mathrm{El} \mathrm{pH}$ alcanzo altos valores al inicio del experimento, presentando pequeñas oscilaciones. Su promedio global fue de 6.4 (d.p=0.19). La sobrevivencia en los tratamientos fue alta. En el experimento con concentraciones de $0.46 \mathrm{mg}$ de $\mathrm{NH}_{3} / 1$ se registró una 
mortalidad de $13 \%$ de los peces en los primeros 20 días y $6.7 \%$ en los últimos 40 días, siendo la sobrevivencia final de $80 \%$, mientras que en el otro experimento de $0.07 \mathrm{mg}$ de $\mathrm{NH}_{3} / 1$, así como en el control, no se observó mortalidad.

Colossoma macropomum mostró que su crecimiento se vio muy poco afectado cuando fue expuesto a las dos concentraciones de amoníaco en las pruebas de los 60 días, y además no fue significativamente diferente (ANOVA; $\mathrm{N}=28 ; \mathrm{GL}=2.2 ; \mathrm{F}=8.87$; $\mathrm{p}=0.11$ ).

Los peces en todos los tratamientos crecieron un promedio de $0.1 \%$ por día ( $\mathrm{D} . \mathrm{P}=0.11$ ). Las diferencias de peso entre los peces no tuvieron interferencia significativa sobre el crecimiento (ANCOVA; $\mathrm{N}=28 ; \mathrm{GL}=1 ; \mathrm{F}=26 ; \mathrm{p}=0.37$ ).

Figura 1. Relación entre la tasa de crecimiento específico (\%/día) y la concentración de $\mathrm{NH}_{3}(\mathrm{mg} / \mathrm{l})$ en los 3 tratamientos.

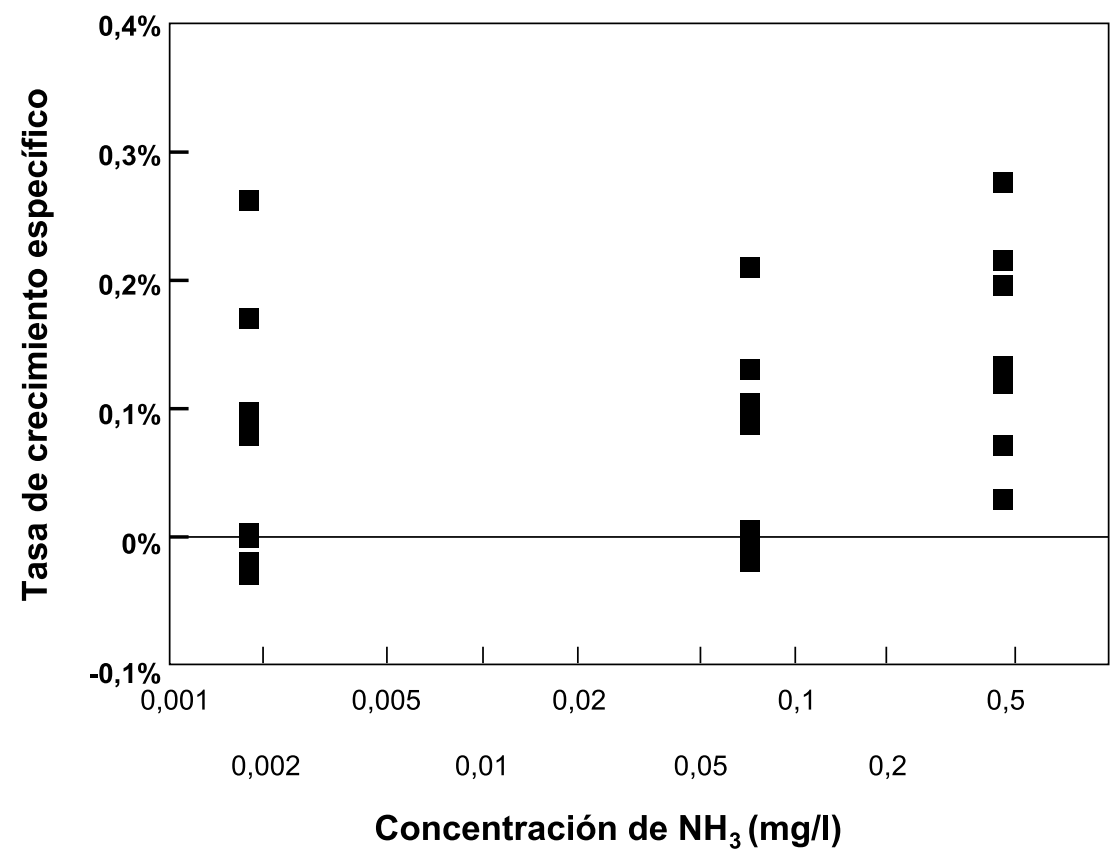




\section{DISCUSIÓN Y CONCLUSIONES}

Valores de amoníaco $\left(\mathrm{NH}_{3}\right)$ superiores a $0.2 \mathrm{mg} / \mathrm{l}$ fueron observadas en cultivos de Ictalurus punctatus (Bader \& Grizzle, 1992) y Colossoma macropomum (Merola y Pagan-Font, 1988; Zaniboni, 1995). La cantidad máxima permisible de amoníaco para peces marinos es de $0.05 \mathrm{mg} / \mathrm{l} \mathrm{de} \mathrm{NH}_{3}$ (Wajsbrot et al., 1993). Los peces adultos son en general menos sensibles que los peces juveniles (Sprague, 1990).

Los peces parecen tener sensibilidad al amoníaco. Thurston et al., (1986) observaron modificaciones en el comportamiento de Pimephales promelas, en concentraciones de $0.928 \mathrm{mg} / \mathrm{l} \mathrm{de} \mathrm{NH}_{3}$. En Ictalurus punctatus estas alteraciones suceden a $0.312 \mathrm{mg} /$ 1 de $\mathrm{NH}_{3}$ (Bader \& Grizzle, 1992), mientras que en otras especies concentraciones inferiores a $0.1 \mathrm{mg} / \mathrm{l} \mathrm{NH}_{3}$ eran suficientes para causar alteraciones crónicas (Broderius et al; Bulkhalter \& Kaya, 1977).

En cuanto al crecimiento de algunas especies de peces, Miranda et al., (1995) demostraron que Mugil platanus presenta una reducción significativa en el crecimiento, en concentraciones superiores a $0.054 \mathrm{mg} / 1 \mathrm{de} \mathrm{NH}_{3}$ e Ictalurus punctatus retarda el crecimiento y presenta degradación en las branquias a $0.12 \mathrm{mg} / \mathrm{l}$ (Piper et al., 1982). Solea solea y Scophthalmus maximus no crecen en aguas con tenores de amoníaco $\left(\mathrm{NH}_{3}\right)$ superiores a $0.38 \mathrm{mg} / \mathrm{l}$ (Alderson, 1979), mientras que Sparus aurata retardo su crecimiento en $68 \%$, expuestos a $0.47 \mathrm{mg} / 1$ de $\mathrm{NH}_{3}$ (Wajsbrot et al., 1993). Pimeplales promelas es el más resistente al amoníaco y presenta reducción en el crecimiento a concentraciones superiores de $0.91 \mathrm{mg} / \mathrm{l}$ (Thurston et al., 1986).

En el presente estudio no se detectó con claridad una inhibición del crecimiento en los peces debido a las concentraciones de amoníaco $\left(\mathrm{NH}_{3}\right)$. Los peces del tanque control donde la concentración era $0.002 \mathrm{mg} / \mathrm{l}$ crecieron en forma semejante a aquellos de los tanques con tóxicos y donde el amoníaco era por lo menos 35 veces más concentrado.

En general, el crecimiento de gamitana en todo los tanques experimentales fue bajo, siendo inferior a lo encontrado en tanques de tierra usados en piscicultura (Campos, 1994). Es posible que otros factores experimentales, que no sea amoníaco, estén interfiriendo y retardando el crecimiento de los peces. Tasas bajas de crecimiento también fueron registradas por Mori (1993), trabajando en un sistema semejante al usado en este estudio. Podría ser también que el tamaño del tanque haya afectado los resultados, en este caso, el efecto del amoníaco sobre el crecimiento podría estar disimulado. 
Experimentos o situaciones donde los peces son transportados de su lugar de origen al laboratorio y las concentraciones de amoníaco en el agua sean inferiores a $0.4 \mathrm{mg} /$ $1 \mathrm{NH}_{3}$ posiblemente no comprometerán la salud de los peces por períodos de 2 meses.

De acuerdo a los resultados obtenidos se concluye que la gamitana es muy resistente al amoníaco $\left(\mathrm{NH}_{3}\right)$ estando entre las especies mas tolerantes a este tóxico.

\section{BIBLIOGRAFÍA}

BADER, J.A.; GRIZZLE, J.M. (1992). Effects of ammonia on growth and survival of recently hatched channel catfish. J. Aquatic. Animal Healt. 4, 17-23.

BRODERIUS, S; DRUMMOND, R; FIANDT, J.; RUSSON, C. (1985). Toxicity of ammonia to early stages of small mouth bass at four ph levels. Environ. Toxic. And chem 4, 87-96.

BULKHALTER, D.E.; KAYA, C. M. (1977). Effects of prolonged exposure to ammonia on fertilized eggs and sac fry of rainbow trout (Salmo gairdneri). Trans. Am. Fish. Soc. 106,470-475.

CAMPOS, B. L. (1994). The culture of gamitana (Colossoma macropomum, Cuvier, 1818) in Latin American. Master's thesis, Southern Illinois University (Carbondale).

ELLIOTT, J.M.; HURLEY, M. A. (1995). The functional relationship between body size and growth rate in fish. Functional Ecology 9, 625-627.

JOBLING, M. (1994). Fish bioenergetics. Chapman \& Hall, London.

MEROLA, N.; PAGÁN-FONT, F. (1988). Pond culture of the Amazon fish tambaqui, Colossoma macropomum: A pilot study. Aquaculture Engineering 7, 113125.

MIRANDA-FILHO, K. C; WASIELESKY-JR, W.; MAÇADA,A. P. (1995). Efeito da amônia e nitrito no crescimento da tainha Mugil platanus (Pisces, Mugilidae). Revista Brasileira de Biologia 55, 45-50. 
MORI, L. A. (1993). Estudo da possibilidade de substitução do fubá de milho (Zea mays) pôr farinha de pupunha (Bactris gasipaes ), em rações de alevinos de tambaqui (Colossoma macropomum) Disertação de mestrado INPA/FUA.

PIPER, R. G; MCELWAIN, I. B; OLME, L. E; MCCRAREN, J. P; FOWLER, L. G.; LEONARD, J. R. (1982). Fish hatchery managment. United States Departament of the Interior. Fish and Wildlife Service, Washington.

SOUSA, R. J.; MEADE, T. L. (1977). Influence of ammonia on the oxigen delivery system of coho salmon hemoglobin. Comparative Biochemestry and Physiology 58, 23-58.

SPRAGUE, J. B. (1990). Aquatic toxicology. In Methods for fish biology, pp. 491527. American Fisheries Society, Bethesda.

THURSTON, R. V; RUSSO, R. C; MEYN, E. L; ZAJDEL, R. K.; SMITH, C. E. (1986). Chronic Toxicity of Ammonia to Fathead Minnows. Transactions of the American Fisheries Society. 115, 196-207.

TOMASSO, J. R; GOUDIE, C. A; SIMCO, B. A.; DAVIS, K. B. (1980). Effects of enviromental $\mathrm{pH}$ and calcium on ammonia toxicity in channel catfish. Transactions of American Fisheries Society. 109, 229-234.

WAJSBROT, N; GASITH, A; DIAMANT, A.M.; POPPER, M, D. (1993). Chronic toxicity of ammonia to juvenile gilthead seabream Sparus aurata and related histopatological effects. Journal of Fish Biology. 42, 321-328.

WOOD, C. M. (1993). Ammonia and Urea Metabolism and Excretion. In the Physiology of fishes ( ed. D. H. Evans), pp. 379-419. CRC Press Inc; Boca Ranton.

ZANIBONI FILHO (1992). Incubação, larvicultura e alevinagem do tambaqui (Colossoma macropomum Cuvier, 1818), tese de doutorado, UFSC, SP. 